\title{
Phase maps for networks of polycrystalline human biological liquids: Statistical and fractal analyses
}

\author{
Yu.O. Ushenko, V.V. Istratiy, V.O. Balanets'ka, D.O. Kvasniyk, V.T. Bachinsky, O.I. Olar \\ Yuri Fedkovych Chernivtsi National University, \\ 2 Kotsybynsky str., 58012 Chernivtsi, Ukraine
}

\begin{abstract}
Performed in this work are complex statistical and fractal analyses of phase properties inherent to birefringence networks of liquid crystals consisting of opticallythin layers prepared from synovial fluid taken from human joints. Within the framework of a statistical approach, the authors have investigated values and ranges for changes of statistical moments of the 1-st to 4-th orders that characterize coordinate distributions for phase shifts between orthogonal components of amplitudes inherent to laser radiation transformed by synovial fluid layers for human joints with various pathologies. Using the Gramm-Charlie method, ascertained are correlation criteria for differentiation of phase maps describing pathologically changed liquid-crystal networks. In the framework of the fractal approach, determined are dimensionalities of self-similar coordinate phase distributions as well as features of transformation of logarithmic dependences for power spectra of these distributions for various types of human joint pathologies.
\end{abstract}

Keywords: birefringence, liquid crystal, statistical moment, phase map.

Manuscript received 10.02.10; accepted for publication 08.07.10; published online 30.09.10.

\section{Introduction}

Among the methods for optical diagnostics of human biological tissues (BT), the methods of laser polarimetric diagnostics aimed at their optically-anisotropic structure are widely spread [1 - 33]. The main "information product" of these methods is availability of coordinate distributions for azimuths $\alpha(x, y)$ and ellipticity $\beta(x, y)$ of polarization (polarization maps) with the following types of analyses: statistical (statistical moments of the 1-st to 4-th orders [13]), correlation (auto- and joint correlation function [14]), fractal (fractal dimensionalities [15]), singular (distributions of amounts of linearly and circularly polarized states [16 - 19]), wavelet analysis (sets of wavelet coefficients for various scales of biological crystals [20, 21]). As a result, interrelations between the set of these parameters and distributions of optical axis directions as well as values of the birefringence characterizing the network of optically uniaxial protein (myosin, collagen, elastin, etc.) fibrils in optically anisotropic components of BT layers can be determined. Using this base, developed is a set of methods for early recognition and differentiation of pathological changes in BT structures related with their degenerative-dystrophic and oncological changes [22, 23].
It is noteworthy that there exists a widespread group of optically anisotropic biological objects for which the methods of laser polarimetric diagnostics are not so efficient. Optically-thin (coefficient of extinction $\tau \leq 0.1$ ) layers of various biological liquids (bile, urine, liquor, synovial liquid, blood plasma, etc.) can be related to these objects. All these layers possess considerably less optical anisotropy of the biological component matter as compared with birefringent BT structures [1]. As a consequence, these objects weakly modulate polarization of laser radiation $\left(\left\{\begin{array}{l}\alpha(x, y) \approx \text { const; } \\ \beta(x, y) \rightarrow 0 .\end{array}\right)\right.$ On the other hand, the biological liquids are more available for a direct laboratory analysis as compared to traumatic methods of BT biopsy. From the above reasoning, it seems topical to search new, additional parameters for laser diagnostics of optically anisotropic structures in biological liquids.

Our work is aimed at searching the possibilities to perform diagnostics of structures inherent to liquidcrystal networks of synovial liquid taken from human joints with various pathologies by using the method to determine the coordinate distributions of phase shifts (phase maps) between orthogonal components of laser radiation amplitudes with the following statistical, correlation and fractal analyses of these distributions. 


\section{Optical model of synovial liquid}

As a base for modeling the optical properties of synovial liquid we use the conception of anisotropy observed in BT protein networks developed in $[1,16,17,24,28,29]$ :

- synovial liquid can be considered as a twocomponent amorphous-crystalline structure;

- $\quad$ optically isotropic is the homogeneous complex of hyaluronic acid with proteins, high amount of leukocytes, high content of whole protein and lactic acid on the background of a low glucose content;

- optically anisotropic - luquid-crystalline phase consisting of a set of optically uniaxial birefringent liquid crystals of various types: fibrin fibers, collagen fibers.

The optical properties of amorphous $\{A\}$ and crystalline $\{C\}$ components of biological liquids can be exhaustively described using the following Jones operators [24]:

$\{A\}=\left\|\begin{array}{ll}a_{11} & a_{12} \\ a_{21} & a_{22}\end{array}\right\|=\left\|\begin{array}{cc}\exp (-\tau l) & 0 \\ 0 & \exp (-\tau l)\end{array}\right\| ;$

(1)

$$
\begin{aligned}
& \{C\}=\left\|\begin{array}{ll}
c_{11} & c_{12} \\
c_{21} & c_{22}
\end{array}\right\|= \\
& \left\|\begin{array}{lc}
\cos ^{2} \rho+\sin ^{2} \rho \exp (-i \delta) ; & \cos \rho \sin \rho[1-\exp (-i \delta)] ; \\
\cos \rho \sin \rho[1-\exp (-i \delta)] & \sin ^{2} \rho+\cos ^{2} \rho \exp (-i \delta) ;
\end{array}\right\| .
\end{aligned}
$$

Here, $\tau$ is the absorption coefficient for laser radiation in the biological liquid layer with the geometric thickness $l ; \rho$ - direction of the optical axis; $\delta=2 \pi / \lambda \Delta$ nd - phase shift between the orthogonal components $E_{x}$ and $E_{y}$ of the amplitude of illuminating laser light with the wavelength $\lambda ; \Delta n$ index of birefringence.

The Jones matrix of the biological liquid layer, where isotropic and anisotropic creations lie in one plane, can be expressed as a sum of operators $\{A\}$ and $\{C\}$

$$
\{M\}=\{A\}+\{C\}=\left\|\begin{array}{ll}
a_{11}+c_{11} ; & a_{12}+c_{12} ; \\
a_{21}+c_{21} ; & a_{22}+c_{22}
\end{array}\right\|,
$$

Let us consider the process of transformation of the complex amplitude $(E \rightarrow U)$ of a laser wave that passed through the biological liquid layer $(\{M\})$ located between two crossed phase filters - quarter-wave plates $\left(\left\{\Phi_{1}\right\}\right.$ and $\left.\left\{\Phi_{2}\right\}\right)$ and polarizers $\left(\left\{P_{1}\right\}\right.$ and $\left.\left\{P_{2}\right\}\right)$, planes of transmission for which make $+45^{0}$ and $-45^{0}$ angles with axes of the highest velocity. The amplitude $U$ of the transformed laser beam in this experimental setup can be determined from the following matrix equation

$$
U=0.25\left\{P_{2}\right\}\left\{\Phi_{2}\right\}\{\mathrm{M}\}\left\{\Phi_{1}\right\}\left\{P_{1}\right\} E \text {. }
$$

Here,

$$
\left\{\begin{array}{l}
E=\left(\begin{array}{l}
E_{x} \\
E_{y} \exp \left(-i \delta_{0}\right)
\end{array}\right) ; U=\left(\begin{array}{l}
U_{x} \\
U_{y} \exp (-i \delta)
\end{array}\right) ; \\
\left\{P_{1}\right\}=\left\|\begin{array}{cc}
1 & 1 \\
1 & 1
\end{array}\right\| ;\left\{P_{2}\right\}=\left\|\begin{array}{cc}
1 & -1 \\
-1 & 1
\end{array}\right\| ; \\
\left\{\Phi_{1}\right\}=\left\|\begin{array}{ll}
1 & 0 \\
0 & i
\end{array}\right\| ;\left\{\left\{\Phi_{2}\right\}=\left\|\begin{array}{ll}
i & 0 \\
0 & 1
\end{array}\right\| ;\right.
\end{array}\right.
$$

In the special case of a plane-polarized wave $E\left(E_{x}=E_{y} ; \delta_{0}=0\right)=\left(\begin{array}{l}1 \\ 1\end{array}\right)$, Eq. (4) acquires the look $U=0.25\left\|\begin{array}{cc}1 & -1 \\ -1 & 1\end{array}\right\| \mid \begin{array}{ll}i & 0 \\ 0 & 1\end{array} \| \times$

$\times\left\|\begin{array}{cc}\cos ^{2} \rho+\sin ^{2} \rho \exp [-i \delta] & \cos \rho \sin \rho\{1-\exp [-i \delta]\} \\ \cos \rho \sin \rho\{1-\exp [-i \delta]\} & \sin ^{2} \rho+\cos ^{2} \rho \exp [-i \delta\end{array}\right\| \times$

$\times||$\begin{tabular}{ll||l||l}
1 & 0 & 1 & 1 \\
0 & $i$
\end{tabular}$\left|\begin{array}{ll}1 & 1\end{array}\right|\left(\begin{array}{l}1 \\
1\end{array}\right)$.

The solution of the matrix equation (6) is the value of complex amplitude $U(\delta)$ that is determined exclusively by the phase shift $\delta$ and does not depend on orientation of the optical axis $\rho$ for a laser image of biological liquid. Being based on it, one can write

$I(\delta)=U U^{*}=I_{0} \sin ^{2}[\delta / 2\rfloor$.

Here, $I_{0}$ is the intensity of a probing laser beam, $I_{\delta}(r)$ - intensity of the laser image for the biological liquid layer in the point $(r)$.

Interrelations (4) to (7) define the algorithm for direct experimental measuring the coordinate distribution of phase shifts $\delta(r)$ between orthogonal components of the amplitudes $U_{x}, U_{y}$ in the laser image of an optically anisotropic biological liquid layer.

\section{Optical scheme and method for experimental measuring the phase maps of biological liquids}

Shown in Fig. 1 is the optical scheme for phasometry of laser images obtained for biological liquids [7, 11,32].

Illumination was carried out using the parallel beam $\left(\varnothing=10^{4} \mu \mathrm{m}\right)$ of He-Ne laser $1(\lambda=0.6328 \mu \mathrm{m})$. Using the polarization illuminator (quarter-wave plate 3 and polarizer 4 ), we formed the beam linearly polarized with the azimuth $45^{\circ}$

The axis of the highest velocity of the quarter-wave plate 5 was oriented at the angle $\Theta=45^{\circ}$ relatively to the transmission plane of the polarizer 4 . Images of biological liquid layers 6 were projected using the micro-objective 7 into the plane of the light-sensitive 


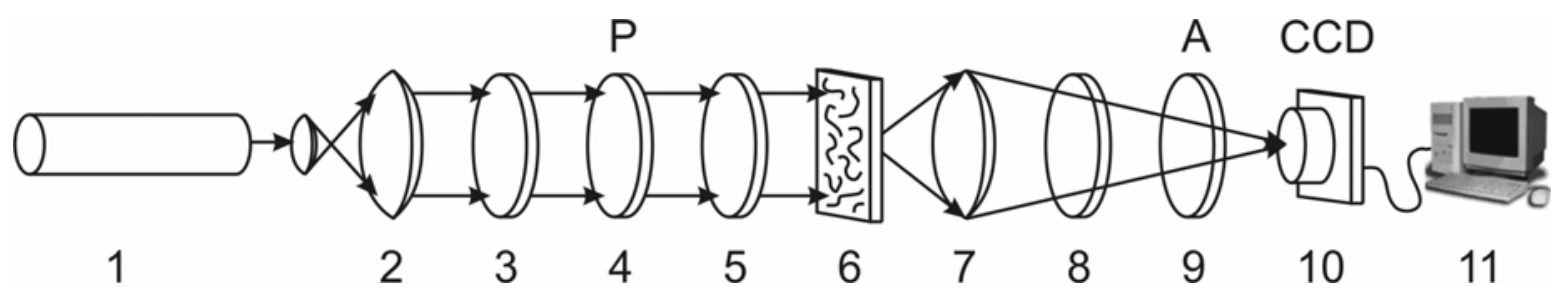

Fig. 1. Optical scheme of the phase meter. 1 - He-Ne laser; 2 - collimator; 3, 5, 8-quarter-wave plates; 4, 9 -polarizer and analyzer, respectively; 6 - investigated object; 7 - micro-objective; 10 - CCD camera; 11 - personal computer.

area $(m \times n=800$ pix $\times 600$ pix $)$ of a CCD camera 10 . Rotating the transmission plane of the analyzer 9 by the angle $\Theta=45^{\circ}$ relatively to the axis of the highest velocity of the quarter-wave plate 8 , formed were the conditions for transmission of left-circular polarized oscillations of points in laser images of biological liquids. The intensity of these oscillations $I_{\delta}$ was registered by each separate pixel in the CCD camera 10 . Thus, we obtained the two-dimensional distribution $I_{\delta}\left(\begin{array}{ccc}r_{11} & , & r_{1 n} \\ r_{m 1} & , & r_{m n}\end{array}\right)$ for this intensity. Further, in accord with (7), we calculated coordinate distributions (phase maps) for phase shifts $\delta(m \times n)$ between orthogonal components of the amplitude for laser images of biological liquids.

\section{Algorithms for statistical, correlation and fractal analyses of phase maps}

To estimate $\delta(m \times n)$ distributions, we calculated the set of statistical moments of the 1-st to 4-th orders $Z_{j=1 ; 2 ; 3 ; 4}$ $[13,18,22,28,30,31]$

$Z_{1}=\frac{1}{N} \sum_{i=1}^{N}\left|\delta_{i}\right|, \quad Z_{2}=\sqrt{\frac{1}{N} \sum_{i=1}^{N} \delta_{i}^{2}}, \quad Z_{3}=\frac{1}{Z_{2}^{3}} \frac{1}{N} \sum_{i=1}^{N} \delta_{i}^{3}$ $Z_{4}=\frac{1}{Z_{2}^{2}} \frac{1}{N} \sum_{i=1}^{N} \delta_{i}^{4}$

As a base for the analysis of the coordinate structure of $\delta(m \times n)$, we took the autocorrelation method using the function [14]

$$
K_{i=1 \div n}(\Delta m)=\lim _{m \rightarrow 0} \frac{1}{m} \int_{1}^{m}\left[\delta_{i}(m)\right]\left[\delta_{i}(m-\Delta m)\right] d m \text {. }
$$

Here, $(\Delta m=1 p i x)$ is the step of changing the coordinate $(x=1 \div m)$ of a distribution in the set of polarization distributions $\delta(m)$ for a specific $i$-th line. The overall expression for the autocorrelation function is obtained by averaging the expression (9) over all the lines $i=1 \div n$
$K(\Delta m)=\frac{\sum_{i=1}^{n} K_{i}(\Delta m)}{n}$

As correlation parameters that characterize the dependences $K(\Delta m)$, we used:

- $\quad$ correlation area $S$

$S=\int_{1}^{m} K(\Delta m) d m$

- correlation moments $Q_{2}$ and $Q_{4}$ that determine the dispersion and excess of the Gramm-Charlie expansion for the autocorrelation function $K(\Delta m)$

$Q=\frac{\sum_{i=1}^{N}(K(\Delta m))_{i}^{4}}{\sum_{i=1}^{N}(K(\Delta m))_{i}^{2}}$

The fractal analysis of $\delta(m \times n)$ distributions was performed using the calculation of logarithmic dependences $\log J(\delta)-\log d^{-1}$ for the power spectra $J(\delta)$

$J(\delta)=\int_{-\infty}^{+\infty} \delta \cos 2 \pi v d v$,

where $v=d^{-1}$ are spatial frequencies that are determined by geometrical dimensions $(d)$ inherent to structural elements of laser images for biological liquids.

The dependences $\log J(\delta)-\log d^{-1} \quad$ are approximated using the least-squares method into the curves $V(\eta)$, straight parts of which are used to determine the slope angles $\eta$ and fractal dimensionalities $F$ in accord with the relation [26] $F=3-\operatorname{tg} \eta$.

Classification of coordinate $\delta(m \times n)$ distributions is fulfilled using the following criteria $[1,14,27,29$, 33]:

- $\quad$ they are fractal with the proviso that the slope angle is constant $(\eta=$ const $)$ for $2-3$ decades of changing the sizes $d$; 
- they are multi-fractal on the condition that there available are several slope angles $V(\eta) \mathrm{p}$;

- $\quad$ they are random, if any stable slope angles $V(\eta)$ do not exist within the whole range of changing the sizes $d$.

In the latter case, the $\log J(\delta)-\log d^{-1}$ distributions are characterized with the dispersion

$$
D=\sqrt{\frac{1}{N} \sum_{i=1}^{N}\left[\log J(\delta)-\log d^{-1}\right]_{i}^{2}} .
$$

\section{Statistical, correlation and fractal parameters of phase maps for synovial liquid}

As objects for experimental studying, we chose optically-thin layers of synovial liquid taken from a joint of the healthy patient (Fig. 2a)and with osteoarthritis (Fig. 2b).

The images of layers prepared from synovial liquid taken from human joints (Fig.2 (a), (d)) are indicative of availability of two fractions - optically isotropic and liquid-crystal network (anisotropic one). As seen, geometric structure and sizes of separate elements in the polycrystalline network of the samples prepared from biological liquids are individual for different types of pathology.

Shown in Fig. 2 are the phase maps (fragments (b), (e)) and histograms (fragments (c), (f)) for distributions of random values inherent to the phase shifts $\delta$ between orthogonal components of the laser radiation amplitude transformed inside layers of synovial liquid taken from healthy patient's joints (fragments (b), (c)) and with osteoarthritis (fragments (e), (f)).

The obtained data show that the value of phase shifts $\delta$ for laser radiation transformed inside layers of synovial liquid lies within the short range of changes $0 \leq \delta \leq 1$. The weak phase modulation is related with two factors. First, it is low geometric thickness $(d=10 \ldots 15 \mu \mathrm{m})$ of the samples. Second, it is weak birefringence $\left(\Delta n \sim 10^{-4} \ldots 10^{-2}\right)$ of liquid-crystal structures in synovial liquid, which is determined by the concentration of fibrin and collagen structures [1].

Our comparative analysis of histograms for distributions of random values inherent to phase shifts $\delta$ in laser images of both types synovial liquids revealed availability of two dominant extreme ranges: $0 \leq \delta \leq 0.15$ and $0.85 \leq \delta \leq 1$. In our opinion, these features of probabilistic phase distributions are related with the influence of optically isotropic $(\delta \rightarrow 0)$ and liquid-crystal $(\delta \rightarrow 1)$ components in the composition of biological liquid.

Being aimed at more specific investigation of phase features for both fractions, we used the following method to select information. From the available coordinate set of values $\delta(m \times n)=\left(\begin{array}{cc}\delta_{11}, \ldots & \delta_{1 n} \\ \delta_{n 1}, \ldots & \delta_{m n}\end{array}\right)$ in phase maps (Fig. 2 (b) and (e)), we found samples of extreme values $\delta(m \times n)=0$ and $\delta(m \times n)=1$. In what follows, by scanning along the direction $x=1 \div n$ we carried out calculation of the amount of extreme values for phase shifts within the column $m=n \times 1$ pix. Within the limits of each local sample $\left.\left(1_{p i x} \times n_{p i x}\right)^{(k=1,2,} \quad 2, m\right)$, we computed the amount $(N)$ of extreme values $\delta(k)=0\left(N_{\min }^{(k)}\right)$ and $\delta(k)=1\left(N_{\max }^{(k)}\right)$. Thus, we found the dependences

$N_{m s n}(x) \equiv\left(N_{m s n}{ }^{(1)}, \quad N_{\min }{ }^{(2)}, \quad \ldots, \quad N_{\min }{ }^{(m)}\right) \quad$ i $N_{\max }(x) \equiv\left(N_{\max }{ }^{(1)}, \quad N_{\max }{ }^{(2)}, \ldots, \quad N_{\max }{ }^{(m)}\right) \quad$ for the amount of extreme values of phase shifts within the limits of laser image for synovial liquid.

Fig. 2 show a set of coordinate distributions $\delta(m \times n)=0 ; 1 \quad$ (fragments $(\mathrm{g}),(\mathrm{k})$ and $(\mathrm{o}),(\mathrm{s}))$ for the dependences of the amount of extreme values $N_{\text {min;max }}(x)$ (fragments (h), (l) and (p), (t)), autocorrelation functions $K_{\min ; \max }(\Delta x)$ (fragments (i), (m) and (q), (u)) and logarithmic dependences $\log J\left(N_{\min ; \max }\right)-\log d^{-1}$ for power spectra of distributions $N_{\text {min;max }}(x)$ (fragments (j), (n) and (r), (v)) that characterize phase maps for the samples of synovial liquid belonging to a healthy patient (fragments $(\mathrm{g})-(\mathrm{j})$ and (o)-(r)) and a patient with osteoarthritis (fragments (k)-(n) and (s)-(v)).

The comparative analysis of the obtained set of experimental data about statistical, correlation and fractal structures in dependences for the amount of extreme values $N_{\min ; \max }(x)$ inherent to phase maps describing layers of synovial liquids taken from joints of a healthy patient and that sick with osteoarthritis enabled to found:

- tendency to a decreasing (increasing) total amount of extreme values $\delta_{\min } \rightarrow 0 \quad\left(\delta_{\max } \rightarrow 1\right)$ of the phase shifts in laser images of layers prepared from synovial liquid of a patient with osteoarthritis (Fig. 2 fragments (h), (l) and (p), (t));

- fact that autocorrelation functions $K_{\min }(\Delta x)$ (Fig. 2 , fragments $(\mathrm{i}, \mathrm{m})$ ) monotonically drop with increasing the step of scanning $\Delta x$ in dependences $N_{\text {min }}(x)$;

- correlation structure of the distribution for the extreme sample $\delta(m \times n)=1$ in the phase map describing the polycrystalline component in synovial liquid of a sick patient changes: at the background of monotonic drop there arise oscillations of values in the dependence $K_{\max }(\Delta x)$ (see Fig. 2, fragment (u)); 


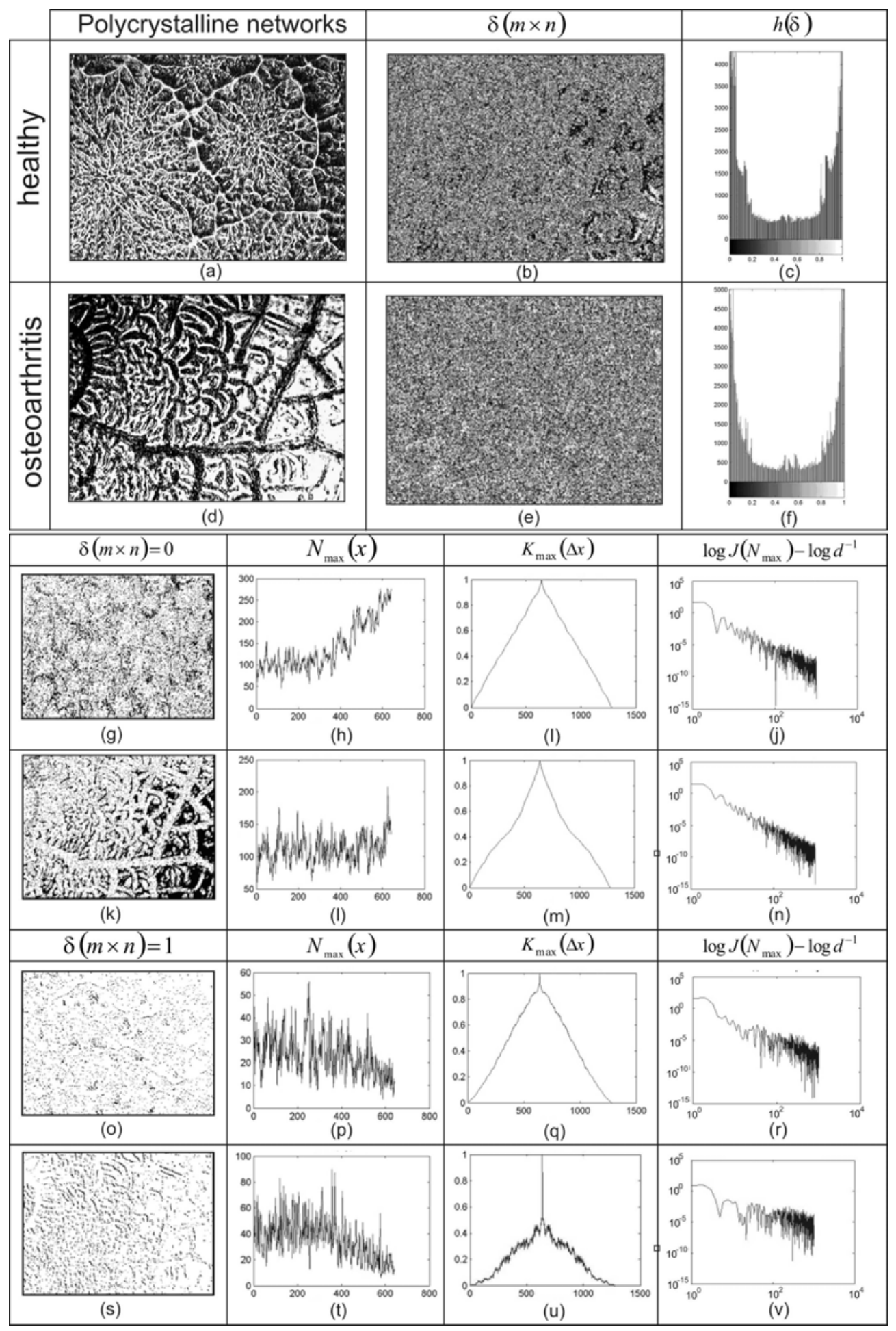

Fig. 2. Here: polycrystalline networks of synovial liquid taken from a joint of the healthy patient (a) and with osteoarthritis (d); coordinate (b, e) and quantitative (c, f) distributions $\delta$ of laser images for the samples of synovial liquid taken from healthy patient's joints $(\mathrm{b}, \mathrm{e})$ and with osteoarthritis arthritis $(\mathrm{c}, \mathrm{f})$; coordinate $(m \times n)(\mathrm{g}, \mathrm{k})$, quantitative $N_{\min }(x)$ (h, l), correlation $K_{\min }(\Delta x)(\mathrm{i}, \mathrm{m})$ and fractal $\log J\left(N_{\min }\right)-\log d^{-1}(\mathrm{j}, \mathrm{n})$ parameters of the extreme sample $\delta(m \times n)=0$ for phase maps of the samples of synovial liquid belonging to a healthy patient $(\mathrm{g}, \mathrm{h}, \mathrm{i}, \mathrm{j})$ and a patient with osteoarthritis $(\mathrm{k}, \mathrm{l}, \mathrm{m}, \mathrm{n})$; coordinate $(m \times n)(\mathrm{o}, \mathrm{s}))$, quantitative $N_{\min }(x)(\mathrm{p}, \mathrm{t})$, correlation $K_{\min }(\Delta x)$ (q, u) and fractal $\log J\left(N_{\min }\right)-\log d^{-1}(\mathrm{r}$, v) parameters of the extreme sample $\delta(m \times n)=0$ for phase maps of the samples of synovial liquid belonging to a healthy patient (o, p, q, r) and a patient with osteoarthritis (s, t, u, v). 
Table 2. Statistical moments $M_{i=1-4}^{\delta}$ that characterize the distributions for amounts of extreme values in coordinate distributions $\delta(m \times n)$ of laser images for synovial liquid.

\begin{tabular}{|l|l|l|l|l|}
\hline$\delta(m \times n)$ & \multicolumn{2}{|l|}{$\delta(m \times n)=0$} & \multicolumn{1}{l|}{$\delta(m \times n)=0$} & $\begin{array}{l}\text { Osteoarthritis } \\
(19 \text { patients })\end{array}$ \\
\hline$M_{i=1-4}^{\delta}$ & $\begin{array}{l}\text { Healthy } \\
(18 \text { patients })\end{array}$ & $\begin{array}{l}\text { Osteoarthritis } \\
(19 \text { patients })\end{array}$ & $\begin{array}{l}\text { Healthy } \\
(18 \text { patients })\end{array}$ & $0.36 \pm 0.042$ \\
\hline$M_{1}^{\delta}$ & $0.46 \pm 0.049$ & $0.51 \pm 0.055$ & $0.22 \pm 0.028$ & $0.13 \pm 0.017$ \\
\hline$M_{2}^{\delta}$ & $0.11 \pm 0.015$ & $0.09 \pm 0.011$ & $0.23 \pm 0.03$ & $2.17 \pm 0.27$ \\
\hline$M_{3}^{\delta}$ & $0.21 \pm 0.024$ & $0.15 \pm 0.018$ & $0.89 \pm 0.097$ & $2.86 \pm 0.31$ \\
\hline$M_{4}^{\delta}$ & $0.29 \pm 0.036$ & $0.23 \pm 0.027$ & $0.77 \pm 0.081$ & \\
\hline
\end{tabular}

Table 3. Correlation $R^{\delta} ; S^{\delta} ; Q^{\delta}$ and fractal $F^{\delta} ; D^{\delta}$ parameters that characterize distributions for amounts of extreme values in coordinate distributions $\delta(m \times n)$ for laser images of synovial liquid.

\begin{tabular}{|l|l|l|l|l|}
\hline$\delta(m \times n)$ & $\delta(m \times n)=0$ & $\delta(m \times n)=0$ & $\begin{array}{l}\text { Osteoarthritis } \\
(19 \text { patients })\end{array}$ \\
\hline Parameters & $\begin{array}{l}\text { Healthy } \\
(18 \text { patients })\end{array}$ & $\begin{array}{l}\text { Osteoarthritis } \\
(19 \text { patients })\end{array}$ & $\begin{array}{l}\text { Healthy } \\
(18 \text { patients })\end{array}$ & $0.31 \pm 0.033$ \\
\hline$R_{2}^{\delta}$ & $0.35 \pm 0.037$ & $0.36 \pm 0.039$ & $0.19 \pm 0.022$ & $2.64 \pm 0.27$ \\
\hline$R_{4}^{\delta}$ & $0.11 \pm 0.013$ & $0.09 \pm 0.009$ & $0.89 \pm 0.0994$ & $0.08 \pm 0.015$ \\
\hline$S^{\delta}$ & $0.28 \pm 0.016$ & $0.24 \pm 0.014$ & $0.17 \pm 0.021$ & statistical \\
\hline$F_{1}$ & $2.09 \pm 0.17$ & $1.97 \pm 0.15$ & $1.92 \pm 0.18$ & $0.18 \pm 0.023$ \\
\hline$D^{\delta}$ & $0.18 \pm 0.022$ & $0.21 \pm 0.028$ & $0.27 \pm 0.033$ & \\
\hline
\end{tabular}

- $\quad$ logarithmic dependences for the power spectra of distributions $N_{\min }(x)$ for the optically isotropic component in synovial liquid of both types possess a stable slope angle (Fig. 2, fragments (d, f)) within the whole range of geometric sizes inherent to the laser image registered by the CCD camera (Fig. 1);

- fractal distributions $N_{\max }(x)$ for phase maps of laser images describing the optically anisotropic fraction of synovial liquid taken from a healthy man (Fig. 2, fragment (j)) are transformed into the statistical ones in the case of osteoarthritis: approximating curve in the dependence $\log J\left(N_{\max }\right)-\log d^{-1}$ has no stable slope (Fig. 2, fragment (n)).

From the quantitative viewpoint, the dependences $N_{\min ; \max }(x)$ illustrate statistical $M_{i=1-4}^{\delta}$, correlation $R^{\delta} ; S^{\delta} ; Q^{\delta}$ and fractal $F^{\delta} ; D^{\delta}$ parameters determined within the limits of two patient groups, and they are summarized in Tables 2 and 3.

Our analysis of the parameters determined experimentally has shown that the following parameters are diagnostically sensitive in observation of inflammatory processes

- $\quad$ statistical moments of the third $\left(M_{3}^{\delta}\right)$ and fourth $\left(M_{4}^{\delta}\right)$ orders in distributions for the amount of extreme values $N_{\max }(x)$ of phase shifts $\delta(m \times n)=1$ in laser images for synovial liquids of both types - differences between them reach 2.4 and 4.1 times;

- $\quad$ dispersion $\left(R_{2}^{\delta}\right)$ and excess $\left(R_{4}^{\delta}\right)$ of the GrammCharlie expansion for autocorrelation functions $K_{\max }(\Delta x)$ related to distributions $N_{\max }(x)$ differ by 1.8 and 2.7 times;

- correlation area $S^{\delta}$ for the autocorrelation dependence $K_{\max }(\Delta x)$ of the distribution for the amount of extreme phase shifts in a laser image inherent to joint synovial liquid of a patient with osteoarthritis is 2.55 times less than that parameter determined for a healthy patient;

- distributions $N_{\max }(x)$ for the phase maps describing synovial liquid for healthy and sick patients are, respectively, fractal and statistical;

- dispersion $D^{\delta}$ of the dependences $\log J\left(N_{\max }\right)-\log d^{-1}$ in the case of pathological changes in the polycrystalline structure of synovial liquid is 1.75 times decreased.

\section{Conclusions}

Thus, one can draw the following conclusions:

1. Synovial liquid of human joints, independently of their physiological state, contains phase-modulating optically anisotropic network of biological crystals.

2. Ascertained and grounded is a set of criteria for phase diagnostics of inflammatory processes (osteoarthritis, atrophic arthritis) as being based on 
statistical (statistical moments of the first to fourth orders), correlation (statistical moments for the GrammCharlie expansion, correlation area) and fractal (fractal dimensionality and dispersion for the distribution of extrema in $\log -\log$ dependences of power spectra) analyses of phase distributions in laser images of synovial liquid.

\section{References}

1. Gang Yao. Two-dimensional depth-resolved Mueller matrix characterization of biological tissue by optical coherence tomography / Gang Yao, Lihong V. Wang // Opt. Lett. - 1999. - Vol. 24. P. 537-539.

2. Wang X. Monte Carlo model and single-scattering approximation of polarized light propagation in turbid media containing glucose / X. Wang, G. Yao, L. - H. Wang // Appl. Opt. - 2002. - Vol. 41. - P. 792-801.

3. Wang $X$. Propagation of polarized light in birefringent turbid media: a Monte Carlo study / X. Wang, L. - H. Wang // J. Biomed. Opt. - 2002. Vol. 7. - P. 279-290.

4. Shuliang Jiao. Depth-resolved two-dimensional Stokes vectors of backscattered light and Mueller matrices of biological tissue measured with optical coherence tomography / Shuliang Jiao, Gang Yao, Lihong V. Wang // Appl. Opt. - 2000. - Vol. 39. P. 6318-6324.

5. Shuliang Jiao. Two-dimensional depth-resolved Mueller matrix of biological tissue measured with double-beam polarization-sensitive optical coherence tomography / Shuliang Jiao, Lihong V. Wang // Opt. Lett. - 2002. - Vol. 27. - P. 101-103.

6. Wang X. Polarized light propagation through the scattering media: time-resolved Monte Carlo and experiments / X. Wang, L. - H. Wang, C. - W. Sun, C. C. Yang // J. Biomed. Opt. - 2003. - Vol. 8. - P. 608-617.

7. Demos S. G. Optical polarization imaging / S. G. Demos, R. R. Alfano // Appl. Opt. - 1997. -Vol. 36. - P. 150-155.

8. Ducros M. G. Polarization sensitive optical coherence tomography of the rabbit eye / M. G. Ducros, J. F. de Boer, H. E. Huang, L. C. Chao, Z. P.Chen, J. S. Nelson, T. E. Milner, H. G. Rylander // IEEE J. Select. Top. Quant. Electron. - 1999. Vol. 5. - P. 1159-1167.

9. de Boer Johannes F. Two-dimensional birefringence imaging in biological tissue using polarization-sensitive optical coherence tomography / Johannes F. de Boer, Thomas E. Milner, Martin J. van Gemert, John S. Nelson, John S // Proc. SPIE. - 1998. - Vol. 3196. - P. 32-37.

10. J. F. de Boer. Determination of the depth-resolved Stokes parameters of light backscattered from turbid media by use of polarization-sensitive optical coherence tomography / J. F. de Boer, T. E.
Milner, J. S. Nelson // Opt. Lett. - 1999. - Vol. 24. - P. 300-302.

11. Handbook of Optical Coherence Tomography; edited by B.E. Bouma and G.J. Tearney // Polarization-sensitive optical coherence tomography / J. F. de Boer, T. E. Milner, M. G. Ducros, S. M. Srinivas and J. S. Nelson. - Marcel Dekker Inc.: New York, 2002. - P. 237-274.

12. Everett M. J. Birefringence characterization of biological tissue by use of optical coherence tomography / M. J. Everett, K. Shoenenberger, B. W. Colston, L. B. Da Silva // Opt. Lett. - 1998. Vol. 23. - P. 228-230.

13. Alexander G. Ushenko and Vasilii P. Pishak, "Laser Polarimetry of Biological Tissue: Principles and Applications", in Handbook of CoherentDomain Optical Methods: Biomedical Diagnostics, Environmental and Material Science, Vol. 1, pp. 93-138, edited by Valery V. Tuchin, Kluwer Academic Publishers, 2004.

14. A.G. Ushenko, "Stokes-correlometry of biotissues," Laser Physics, vol. 10(5), pp.12861292, 2000.

15. A.G. Ushenko, "The Vector Structure of Laser Biospeckle Fields and Polarization Diagnostics of Collagen Skin Structures," Laser Physics, vol. 10(5), pp. 1143-1149, 2000.

16. A.G. Ushenko, I. Z.Misevich, V. Istratiy, I. Bachyns'ka, A. P. Peresunko, Omar Kamal Numan, and T. G. Moiysuk, "Evolution of Statistic Moments of 2D-Distributions of Biological Liquid Crystal Net Mueller Matrix Elements in the Process of Their Birefringent Structure Changes," Advances in Optical Technologies, vol. 2010, Article ID 423145, 2010.

17. O. V. Dubolazov, A. G. Ushenko, V. T. Bachynsky, A. P. Peresunko, and O. Ya. Vanchulyak, "On the Feasibilities of Using the Wavelet Analysis of Mueller Matrix Images of Biological Crystals," Advances in Optical Technologies, vol. 2010, Article ID 162832, 2010.

18. O.V. Angelsky, A.G. Ushenko, Ye.G. Ushenko, “2D Stokes Polarimetry of Biospeckle Tissues Images in Pre-Clinic Diagnostics of Their Pre-Cancer States," Journal of Holography and Speckle, vol. 2(1), pp.26-33, 2005.

19. A.G. Ushenko, "Laser diagnostics of biofractals," Quantum Electronics, vol. 29(12), pp. 1078-1084, 1999.

20. O.V. Angel'skii, A.G. Ushenko, A.D. Arkhelyuk, S.B. Ermolenko, D.N. Burkovets, "Structure of matrices for the transformation of laser radiation by biofractals," Quantum Electronics, vol. 29(12), pp. 1074-1077, 1999.

21. O.V. Angel'skii, A.G. Ushenko A.D. Arheluk, S.B. Ermolenko, D. N. Burkovets, "Scattering of Laser Radiation by Multifractal Biological Structures," Optics and Spectroscopy, vol. 88(3), pp. 444-448, 2000. 
22. A.G. Ushenko, "Polarization Structure of Biospeckles and the Depolarization of Laser Radiation," Optics and Spectroscopy, vol. 89(4), pp. 597-601, 2000.

23. A.G. Ushenko, "Laser polarimetry of polarizationphase statistical moments of the object field of optically anisotropic scattering layers," Optics and Spectroscopy, vol. 91(2), pp. 313-316 2001.

24. A.G. Ushenko, "Polarization contrast enhancement of images of biological tissues under the conditions of multiple scattering," Optics and Spectroscopy, vol. 91(6), pp. 937-940, 2001.

25. A.G. Ushenko, "Laser probing of biological tissues and the polarization selection of their images," Optics and Spectroscopy, vol. 91(6), pp.932-936, 2001.

26. A.G. Ushenko, "Correlation processing and wavelet analysis of polarization images of biological tissues," Optics and Spectroscopy, vol. 91(5), pp.773-778, 2002.

27. A.G. Ushenko, "Polarization correlometry of angular structure in the microrelief pattern or rough surfaces," Optics and spectroscopy, vol. 92(2), pp.227-229, 2002.

28. Oleg V. Angelsky, Alexander G. Ushenko, and Yevheniya G. Ushenko, "Complex degree of mutual polarization of biological tissue coherent images for the diagnostics of their physiological state," J. Biomed. Opt., vol. 10(6), 060502, 2005.

29. Oleg V. Angelsky, Alexander G. Ushenko, Yevheniya G. Ushenko, Yuriy Y. Tomka, "Polarization singularities of biological tissues images," J. Biomed. Opt., vol. 11(5), 054030, 2006.

30. Alexander G. Ushenko, "Polarization structure of laser scattering fields," Optical Engineering, vol. 34(4), pp. 1088-1093, 1995.

31. A.G. Ushenko, M.T. Strinadko, S.B. Yermolenko, M.A Neduzhko, "High resolution polarization interferometry in surface and layer diagnostics," International journal of optoelectronics, vol. 4(6), pp. 563-574, 1989.

32. O.V. Angelsky, A.G. Ushenko, and Ye.G. Ushenko, "Investigation of the correlation structure of biological tissue polarization images during the diagnostics of their oncological changes,” Phys. Med. Biol., vol. 50, pp. 4811-4822, 2005.

33. O.G. Ushenko, S.G. Guminetsky, A.V. Motrich, "Optical properties of urine, blood plasma and pulmonary condensate of the patients with pulmovnary form of tuberculosis," Fotoelektronika, vol.16, pp. 133-139, 2007. 